\title{
RELATIONS BETWEEN HYPERSURFACE CROSS RATIOS, AND A COMBINATORIAL FORMULA FOR PARTITIONS OF A POLYGON, FOR PERMANENT PREPONDERANCE, AND FOR NON-ASSOCIATIVE PRODUCTS
}

\author{
TH. MOTZKIN
}

This note improves, in two respects, the results of $\$ 3.6$ of my paper The hypersurface cross ratio. ${ }^{1}$ There it is shown that the number $c_{n}$ of independent hypersurface cross ratios that can be formed of $2 n$ forms in $n$ variables is 2 for $n=2,5$ for $n=3$, and 14 for $n=4$. The proof employs the relations between cross ratios obtained by some simple permutations of the forms; let $R$ be the set of these relations. It is remarked that the cross ratios of $2 n-1$ forms in $n$ variables, and of $2 n-1$ forms in $n-1$ variables, are connected by the same relations as the cross ratios of $2 n$ forms in $n$ variables, as far as these are consequences of the relations $R$, a "perhaps void restriction." We now prove that $c_{n}=C_{2 n, n} /(n+1)$, and that the restriction is in fact void, so that a complete knowledge of the relations between the cross ratios of $2 n-1$ forms, of $2 n$ forms, and of $2 n+1$ forms in $n$ variables is obtained. ${ }^{2}$ The corresponding theorems for generalized intersections and one more variable are established at the same time.

The same facts hold for a general class of function ratios, which includes hypersurface cross ratios and generalized intersections as very special cases. The number $c_{n}$ of independent function ratios has a simple combinatorial meaning, and appears also as the number of partitions of a polygon by non-intersecting diagonals into triangles, or of a cyclically arranged set into non-interlaced subsets, as the number of possibilities of never losing majority (in an election or a game $^{8}$ ), and as the number of different products of given terms in a given order, in a non-associative multiplication. For the combinatorial formula, seven proofs are given, six extended to generalizations. ${ }^{4}$

Received by the editors September 16, 1946.

1 Bull. Amer. Math. Soc. vol. 51 (1945) pp. 976-984.

2 For forms of a sufficiently high degree. Cf., on the other hand, for 5, 5 and 6 linear forms in 2, 3 and 3 variables respectively, $\$ \S 3,4,5$ of The pentagon in the projective plane, with a comment on Napier's rule, Bull. Amer. Math. Soc. vol. 51 (1945) pp. 985989.

${ }^{8}$ Or for drops falling on a board one-half of which is supported, and similar physical schemes.

${ }^{4}$ For an eighth proof cf. P. Erdös and I. Kaplansky, Sequences of plus and minus, Scripta Mathematica vol. 12 (1946) pp. 73-75 (for $[f(n, n)]^{2}, \operatorname{read} f(n, n) f(n+1, n+1)$, or permit only diagonal moves; in (4), read $m \leqq n)$. I have made use of oral remarks by $A$. Dvoretzky (in 2.3-2.5) and E. Jabotinsky (in 1.1). 
1. The main theorem. 1.1. Let $F$ be a function of $n$ arguments (variables, points, polymonials) that is (1) almost symmetric ( $F$ becomes $F$ or $-F$ for every permutation of the arguments), and (2) such that the $C_{2 n, n}$ functions of any $n$ from among $2 n$ arguments $a_{11}, a_{12}, a_{21}, a_{22}, \cdots, a_{n 1}, a_{n 2}$ are independent. We then define the function ratio of the $n$ pairs of arguments, in the given order, as $G=\prod F\left(a_{1 r_{1}}, \cdots, a_{n r_{n}}\right) \rho$, where $\rho=(-1)^{\sum r_{i}}$, and the product extends over the $2^{n}$ possible systems $r_{1}, \cdots, r_{n}$. We shall prove:

The number of independent function ratios that can be formed of $2 n$ arguments is $C_{2 n, n} /(n+1)$.

Obviously, the relations between the function ratios do not depend on the particular function, except possibly for the influence of the factor -1 affixed to $F$ and hence to $G$.

1.2. Further, the same number and relations are obtained in two more cases: one of the arguments is allowed to be a symbol $p$ or $q$, defined as follows. For a symbol $p$, every $F$ involving $p$ is \pm 1 , and independence is only supposed to hold for the $C_{2 n-1, n}$ functions of any $n$ from among the remaining $2 n-1$ arguments. For a symbol $q$, again every $F$ involving $q$ is \pm 1 , while in every other factor $F$ of a function ratio, the argument paired with $q$ in the definition of that function ratio is omitted (and possibly replaced by a factor -1 ); here $F$ is a function of $n-1$ arguments, and the $C_{2 n-1, n-1}$ functions of any $n-1$ from among the $2 n-1$ non-symbolic arguments are supposed to be independent.

1.3. In particular, the function $F$ may be the resultant of $n$ forms in $n$ variables, or the intersection of $n$ general hypersurfaces in the projective $n$-space over an algebraically closed field, for forms and hypersurfaces of a sufficiently high degree (if a symbol $q$ occurs, replace every $n$ by $n-1$ ). That in these cases the resultants (and the intersections, considered as resultants with an additional linear form) are independent, will be shown separately. ${ }^{5}$

1.4. We consider the influence on $G$ of three kinds of simple permutations of the arguments. (1) If two arguments of the same pair are interchanged, then $G$ becomes $1 / G$. (2) If two pairs are interchanged, then $G$ becomes $\pm G$. By (1) and (2) there belongs, to every division of the arguments in pairs, essentially one function ratio. (3) If two arguments of one pair and a third argument are permuted cyclically, then the product of the three function ratios obtained is easily seen to be \pm 1 , even if one of the arguments is a symbol $p$ or $q$. It follows that from among the ratios belonging to divisions with $n-2$

\footnotetext{
'In the subsequent note Independence of resultants, Bull. Amer. Math. Soc. vol. 54 (1948) pp. 360-365.
} 
fixed pairs, any one is determined by the two others.

1.5. Let the arguments be represented by $2 n$ points on a circle. A division $D$ in pairs is then symbolized by $n$ chords. It has a number $c$ of crossings (pairs of chords intersecting within the circle). Choose two chords $P$ and $Q$ forming a crossing and express the function ratio of $D$ by the function ratios of the other divisions $D^{\prime}$ and $D^{\prime \prime}$ with the same remaining $n-2$ chords. A chord of $D$ that crosses $P$ and $Q$ crosses either both new chords $P^{\prime}$ and $Q^{\prime}$ of $D^{\prime}$ or neither of them; a chord that crosses just one of $P$ and $Q$ crosses exactly one of $P^{\prime}$ and $Q^{\prime}$, and a chord that crosses neither $P$ nor $Q$ crosses neither $P^{\prime}$ nor $Q^{\prime}$. Hence the function ratio of $D$ can be expressed by the function ratios of divisions with numbers $c^{\prime}$ and $c^{\prime \prime}$ of crossings, less (by an odd number) than $c$, and ultimately by the function ratios of divisions with no crossing. It remains to be shown that the number of these divisions is $C_{2 n, n} /(n+1)$, and that their function ratios are independent.

1.6. The independence of the function ratios belonging to divisions with $c=0$ follows from the independence of the functions $F$, if we show that these function ratios can be so arranged that each involves a function $F$ that occurs in none of the preceding function ratios.

Let $1, \cdots, 2 n$ be the points in their order on the circle. Arrange the sets of $n$ numbers, and the corresponding functions $F$, lexicographically. Among the functions forming a given function ratio there is a last one involving the greater number of every pair. Now arrange the function ratios with $c=0$ according to their last function; it then remains to verify that each of them is determined by its last function.

For a given $F$, let $k$ be the first point involved. In a division whose last function is $F, k$ must be paired with a smaller number, and $k-1$ with a greater number. Hence if $c=0$, then $k-1$ and $k$ must be paired. Omit both, and repeat the procedure.

A symbol $p$ or $q$ should be denoted by 1 . The point paired with $q$ remains till the end of the procedure.

1.7. Of the $N=(2 n) ! / n ! 2^{n}$ divisions of $2 n$ points on a circle into $n$ pairs, $N \cdot 2^{n} /(n+1) !=C_{2 n, n} /(n+1)$ are without crossing.

For let the number of divisions without crossings be $c_{n}$. We wish to express $c_{n+1}$ by $c_{1}, \cdots, c_{n}$. Let $A$ be one of the $2 n+2$ points. The point $A$ must be paired with a point $B$ for which the number of points on either $\operatorname{arc} A B$ is even. If the arcs bear $2 j$ and $2 k$ points, besides $A$ and $B$, then $j+k=n$, and there are $c_{j} c_{k}$ divisions without crossings containing the pair $A B$ (put $c_{0}=c_{1}=1$ ). Hence we have the recursion formula $c_{n+1}=\sum c_{j} c_{k}$. It follows that the series ${ }^{6} y=\sum c_{n} x^{n}$ satisfies

- The series is convergent until $x^{\prime}=0$, that is, for $|x|<1 / 4=\lim c_{n} / c_{n+1}$ (in 2.1 for $\left.|x|<(l-1)^{l-1} / l^{l}\right)$, but we need it only for formal operations. 
the equation $y^{2}=(y-1) / x$, whence $2 x y=1-(1-4 x)^{1 / 2}=1-\sum(1 / 2)$ $\cdot(-1 / 2) \cdots \cdot(3 / 2-n)(-4 x)^{n} / n !=2 \sum C_{2 n-2, n-1} x^{n} / n$, so that $c_{n}=C_{2 n, n} /(n+1)=C_{2 n+1, n} /(2 n+1)$. For large values of $n$ we have $c_{n} \sim 4^{n} n^{-3 / 2} \pi^{-1 / 2}$.

1.8. The same recursion formula holds also for the number $c_{n}$ of partitions of a convex polygon of $n+2$ sides, by non-intersecting diagonals, into triangles. Indeed, a fixed side of a polygon of $n+3$ sides belongs, in a partition as required, to a certain triangle; after choosing the triangle there remain two polygons of $j+2$ and $k+2$ sides, with $j+k=n$, to be divided in the same manner. Here $j=0, \cdots, n$, $c_{0}=1$. Hence, the value of $c_{n}$ is again $C_{2 n, n} /(n+1){ }^{7}$

If, for a regular polygon, congruent or symmetric partitions are considered as equal, then the numbers $1,1,2,5,14,42,132,429$, $1430,4862,16796,58786, \cdots$ are reduced to $1,1,1,1,3,4,12,27$, $82,228,783,2282, \cdots$, which are $\sim c_{n} / 2 n$.

There is no natural one-to-one correspondence between the partitions of a polygon and the divisions with no crossings. For the latter, the numbers of types of congruent or symmetric divisions begin with $1,1,1,2,3,6,12,25, \ldots$ and are $\sim c_{n} / 4 n$. Congruence and symmetry are defined for an equally divided circle. The circle itself is determined by the set of all partitions with no crossing. Indeed, for every pair on the circumference there are $(n-1)^{2}$ pairs that appear together with the given pair in at least one partition, while for a pair that separates $2 j$ from $2 k$ points, $j+k=n-1$, the corresponding number is $j^{2}+k^{2}<(n-1)^{2}$.

1.9. The recursion formula holds also for the number $c_{n}$ of different products of $n+1$ factors, in a given order, in a non-associative multiplication: every product of $n+2$ terms is obtained by multiplying a product of $j+1$ and a product of $k+1$ factors, with $j+k=n, j=0$, $\cdots, n, c_{0}=1$. Hence again $c_{n}=C_{2 n, n} /(n+1) .^{8}$

2. Generalizations. 2.1. To determine the number $c_{n}$ of divisions

${ }^{7}$ This and some more general results, including the formula for $c_{n}$ in 2.2, were obtained in a series of papers from Euler until Cayley, On the partitions of a polygon, Proc. London Math. Soc. vol. 22 (1891) pp. 237-262; Collected Mathematical Papers, vol. 13, pp. 93-113. Cf. the references given in Rowe and Taylor, Note on a geometrical theorem, Proc. London Math. Soc. vol. 13 (1881-1882) pp. 102-106, to which the Catalogue of scientific papers 1800-1900, Royal Society of London, 1908, pp. 86 and 437 adds papers by Binet, Faure, Grunert, Kirkman, and Liouville.

${ }^{8}$ This was perhaps first remarked by Paolina Quarra, Calcolo delle parentesi, Torino Atti vol. 53 (1918) pp. 1044-1047. The connection with the result of Gummer (see footnote 11) was noted by J. H. M. Wedderburn, The functional equation $g\left(x^{2}\right)=2 \alpha x+[g(x)]^{2}$, Ann. of Math. (2) vol. 24 (1923) pp. 121-140, on p. 121. For these references I am indebted to the referee. 
without crossings of $n l$ points on a circle into $n l$-tuples, choose again, for a fixed point from among $(n+1) l$ points, the $l$-tuple containing the point. There remain arcs with $j_{1} l, \cdots, j_{l} l$ points such that $j_{1}+\cdots+j_{l}=n$. For the first arc, there are $c_{j_{1}}$ divisions $\left(c_{0}=1\right)$, and so on. The recursion formula obtained, $c_{n+1}=\sum c_{j_{1}} \cdots c_{j_{j}}$, corresponds to a function $y=\sum c_{n} x^{n}$ with $y^{l}=(y-1) / x$. By Lagrange's formula for implicit functions ${ }^{\theta}$ we have $c_{n}=C_{l n, n} /((l-1) n+1)$ $=C_{l n+1, n} /(l n+1) \cdot{ }^{10}$ For $l=2$, this is a second (less elementary) proof for the evaluation of 1.7 .

2.2. The same recursion formula is obtained for the number of partitions of a convex polygon of $m=(l-1) n+2$ sides, by non-intersecting diagonals, into polygons of $l+1$ sides (polygons with other values of $m$ cannot be so divided). To see this choose, for a fixed side, the adjacent partial polygon, and proceed as before.

Yet, though it might seem so at first sight, $c_{n}$ is not connected with the number of independent generalized function ratios of the form

$$
G=\prod F\left(a_{1 r_{1}}, \cdots, a_{n r_{n}}\right)^{\rho}, \quad r_{j}=1, \cdots, l, \rho=\epsilon^{\sum r_{j}}, \epsilon^{l}=1, l>2 .
$$

The latter number is, even for a fixed division into $l$-tuples, equal to the number of independent ordinary function ratios. Indeed, every generalized function ratio can without difficulty be expressed as a product of powers of ordinary function ratios, and vice versa.

2.3. If the $l n$ points on the circle are denoted in order by $1, \cdots, l n$, then every division without crossing may again be uniquely represented by its last term: the set $N$ comprising the greatest number in every $l$-tuple. As in the case of $l=2$, the smallest number of $N$ forms an $l$-tuple together with the $k=l-1$ preceding numbers of the complementary set $M$, and so on.

Not every set $N$ of $n$ numbers from among $1, \cdots, l n$ is the last set of a division without crossing, for the procedure of omitting the smallest number of $N$, together with its predecessors, cannot always be repeated until no number is left. This can be done if and only if $k$ times the number $n(t)$ of numbers belonging to $N$, from among $1, \cdots, t(t \leqq l n)$, is not more than the number $m(t)$ of numbers belonging to the set $M$ of the $k n$ numbers outside $N$. The number $c_{n}$ can, therefore, be interpreted as the number of possibilities for a candidate $M$ in an election, who ultimately obtains $k$ times as many votes as his adversary $N$, to have, during the whole time of the counting, at least $k$ times as many votes as $N$. Since $1, \cdots, \ln$ con-

${ }^{9}$ Goursat, Cours d'analyse, 5th ed., vol. 1, 1927, p. 427.

${ }^{10}$ Polya and Szegö, Aufgaben und Lehrsätze aus der höheren Analysis, vol. 1, 1925, part II, problem 211 . 
tains $C_{l n, n}$ sets of $n$ numbers, the probability of "perpetual preponderance" is $c_{n} / C_{l n, n}=1 /(k n+1)$. If an additional number 0 is prefixed and $M$ (getting $k n+1$ of $l n+1$ votes) is required always to have more than $k$ times as many votes as $N$, the probability is $c_{n} / C_{l n+1, n}=1 /(\ln +1)$.

2.4. Generally, for $M$ with $m=k n+r$ and $N$ with $n$ votes, we shall prove the probability of the last-mentioned kind of permanent preponderance to be $r / s$, where $s=m+n=l n+r$ is the total number of votes. ${ }^{11}$ This includes a third proof of the special case $k=r=1$.

For any given arrangement of the votes, let $u$ be the smallest number such that $r(t)=m(t)-k n(t)>0$ for every $t>u$. Then $r(u) \leqq 0$, and the vote $u+1$ belongs to $M$ and adds 1 to $r(u)$. Hence $r(u)=0$. Changing the arrangement of the first $u$ votes alters neither this fact nor the characteristic property of $u$. For every fixed $u>0$ there are $k$ times as many arrangements beginning with a vote for $M$ as there are beginning with one for $N$. On the other hand, for every arrangement beginning with a vote for $N$ we have $u>0$. The latter arrangements have the probability $n / s$, hence there remains for $u=0$ the probability $1-n / s=r / s$.

For $k=1$, the first moment of equality of votes may be considered instead of the last moment, which leads to a fourth proof of 1.7.

2.5. The significance of the value $r / s$ is given by the theorem:

Of every s arrangements that are obtained from each other by a cyclic permutation, exactly $r$ show permanent preponderance.

In case not all the $s$ arrangements are different, they fall into periods of different arrangements; every period contains the same number of arrangements with permanent preponderance, so that also $r / s$ of the different arrangements have permanent preponderance.

To prove the theorem (and hence 1.7 for a fifth time), let the votes for $M$ and $N$ be arranged cyclically. Neither a vote $v$ for $N$ nor any of the $k$ preceding votes is the beginning of an arrangement with

${ }^{11}$ For $k=1$ this is the "ballot problem," formulated and solved, by formula (3) of $\$ 2.7$ of this note, by Bertrand, Solution d'un probleme, C. R. Acad. Sci. Paris vol. 105 (1887) p. 369 . Ibid. pp. 407,435 , and 440 , E. Barbier gave, without proof, the probabilities $r / s$ of 2.4, and $r / s$ of 2.6 for the set of all arrangements. Barbier may have proved his first result, and the second one for $s>\ln$, if it holds for $s=\ln$, by the abovementioned formula (3); I do not see how he could prove the second result for $s=l n$.

For further history, and for related theorems, see A. Dvoretzky and Th. Motzkin, $A$ problem of arrangements, Duke Math. J. vol. 14 (1947) pp. 305-313; to the different independent formulations of the ballot problem mentioned there, add P. Franklin, Amer. Math. Monthly vol. 25 (1918) p. 118, problem 2681, solution by C. F. Gummer, ibid. vol. 26 (1919) pp. 127-128, and H. D. Grossman, Scripta Mathematica vol. 12 (1946) pp. 223-225. 
permanent preponderance. From $v$ go backwards until, on the arc covered, the number of votes for $M$ is $k$ times that for $N$, which must happen; for example go as long as the number of votes for $M$ is at most $k$ times that for $N$ : when stopping it will be exactly $k$ times. To every vote $v$ for $N$ there belongs such an $\operatorname{arc} A_{v}$ of forbidden beginnings; and to every vote for $N$ on $A_{v}$ there belongs an arc contained in $A_{v}$. Outside the maximal arcs of forbidden beginnings there remain obviously no votes for $N$ and $r$ votes for $M$. Each of them is good, since the first vote $v$ disturbing the preponderance of $M$ would be for $N$, and $A_{v}$ would include the given beginning. ${ }^{12}$

2.6. It is easy to see (still for $s \geqq l n$ ) that if, from an $\operatorname{arc} A_{v}$, we delete every partial arc $A_{v^{\prime}}, v^{\prime} \neq v$, then there remains only the vote $v$, together with $k$ votes for $M$, and that for each of these votes $v$ is the first vote that disturbs preponderance. Since the deleted votes can be divided into groups of $l$ consecutive votes, the probability $l n / s$ for non-preponderance is equally distributed among the possible remainders, after division by $l$, of the number of the first vote that disturbs preponderance:

Of every $s \geqq$ In arrangements that are obtained from each other by a cyclic permutation there are exactly $n$ for which the number of the first vote disturbing preponderance has a given value $\bmod l$.

2.7. Instead of $r(t)=m(t)-k n(t)$, other functions of two or more non-negative integers $m, n, \cdots$, not all of which are 0 , may be required to remain positive. Let the given function be $m-f(n)$, and let $p_{m, n}$ be the corresponding number of possibilities. Obviously (1) $p_{m, n}=0$ for $m \leqq f(n)$, while for $m>f(n)$, (2) $p_{m, n}=1$ if $m n=0$ and (by classification of the possibilities according to the last vote) (3) $p_{m, n}$ $=p_{m, n-1}+p_{m-1, n}$ if $m n>0$. (Similar equalities hold for more than two variables.) By (1), (2), (3), $p_{m, n}$ is uniquely defined. There is $p_{m, n}=0$ if $m \leqq f\left(n^{\prime}\right), n^{\prime} \leqq n$, so that $f(n)$ may be supposed to be an increasing function. Subtracting a constant from $m$, or adding unity to $m$, we may also suppose $f(0)=0$.

The expression $q_{m, n}=C_{m+n, m}(m-k n) /(m+n)=C_{m+n-1, n}-k C_{m+n-1, m}$ fulfils (3) and $q_{m, 0}=1$. For an integer $k, q_{k n, n}=0$, so that the $p_{m, n}$ belonging to $f(n)=k n$ equals $q_{m, n}$ for $m \geqq k n$, a fourth proof for 2.4 and a seventh proof for 1.7. This proof, the proof in 2.4, and the cycle

12 Another (for 1.7 sixth) proof of the "cycle theorem": The arrangement$u+1, \cdots, s, 1, \cdots, u$ shows permanent preponderance if and only if (1) $r(t)>r(u)$ for every $t>u$, and (2) $r(u)<r(t)+r$ for every $t<u$. Let $q \leqq 0$ be the minimum value of $r(t)$ for $t=0,1, \ldots$. Put $r(u)-q=p$; by (1) and (2) we have $p<r$, and $u$ is the smallest value such that $r(t)>q+p$ for every $t>u$. To every non-negative $p<r$ corresponds a value of $u$; hence there are $r$ such values. 
theorem 2.5 with its two proofs remain valid for preponderance $m-k n-k^{\prime} n^{\prime}-\cdots>0$ over more than one adversary.

For general $f(n)$, the expression $q_{m, n}=a_{0} C_{m+n, m}+a_{1} C_{m+n-1, m}$ $+\cdots+a_{n}$, which fulfils (3), can be made equal to $p_{m, n}$ for $m \geqq f(n)$ by putting $a_{0}=1$ and, after determination of $a_{1}, \cdots, a_{n-1}$, choosing $a_{n}$ such that $q_{m, n}=0$ for the greatest integer $m \leqq f(n)$. The law for the characteristic sequence of integers $a_{0}, a_{1}, \cdots$ is not known even for the simplest functions except $k n$ with integral $k$ (and related functions, as $\max (k n-c, 0)$ or $\min (k n, c), c>0)$.

2.8. Another generalization wanted is the number of mixed partitions, that is, of partitions of a convex polygon by non-intersecting diagonals into polygons of $l$ sides, where $l$ is not restricted to a single value. The simplest case, partition into triangles and quadrangles, has the recursion formula $c_{n+1}=\sum c_{j} c_{n-j}+\sum c_{j} c_{k} c_{n-1-j-k}$ for the number $c_{n}$ of partitions of an $(n+2)$-gon. The corresponding series $y=\sum c_{n} x^{n}$ fulfils the equation $y-1=x y^{2}+x^{2} y^{3}$. The sequence $c_{0}, \cdots$ begins $1,1,3,10,38,154,654,2871,12925,59345, \cdots$, with $c_{n+1} / c_{n} \rightarrow 27 / 5=5.4 .^{13}$

If $l$ is allowed to take all values $3,4, \cdots$, and if $P Q R$ and $P Q R^{\prime}$ are consecutive vertices of the given and of a partial polygon, then classification of the partitions according to the position of $R^{\prime}$ gives the formula $c_{n+1}=b_{1} c_{n}+b_{2} c_{n-1}+\cdots+b_{n+1} c_{0}$ with $c_{0}=1$, where $b_{n+1}$ is the number of partitions with $R=R^{\prime}$. By omission of $Q$ in the latter and possibly connection of $P$ with $R$ we see that $b_{n+1}=2 c_{n}$ for $n>0$, while $b_{1}=1$. Hence we have $c_{n+1}=c_{0} c_{n}+2\left(c_{1} c_{n-1}+\cdots+c_{n} c_{0}\right)$. There follows $(y-1) / x=2 y^{2}-y, 4 x y=x-\sum_{1}^{\infty} C_{1 / 2, n}\left(x^{2}-6 x\right)^{n}$. The values of $c_{0}, \cdots$ are $1,1,3,11,45,197,903,4279,20793,103049,518859$, $\cdots$, with $c_{n+1} / c_{n} \rightarrow 3+2^{3 / 2}=5.83$.

The numbers of types of congruent or symmetric partitions of a regular polygon begin with $1,1,2,3,9,20,73, \cdots$.

2.9. The number $c_{n}$ of divisions of $n$ points on a circle into sets of $l$ points without crossing may also be generalized to different values of $l$, but with other results than for partitions of polygons. For $l=2$ or 3 we are led to $y-1=x^{2} y^{2}+x^{3} y^{3}$. The sequence $c_{n}$ begins $1,0,1,1$, $2,5,8,21,42,96,222,495,1175, \cdots$, with $c_{n+1} / c_{n} \rightarrow 2.61$. For $l=1$ or 2 we have $y-1=x y+x^{2} y^{2}$, and the sequence $c_{n}$ begins $1,1,2,4$, $9,21,51,127,323,835,2188,5798,15511, \cdots$, with a ratio tending to 3 .

If all values $l=1,2, \cdots$ are allowed, then the numbers of types

13 This and the following three limits are obtained as reciprocals of the (unique) absolutely least $x$ with $x^{\prime}=0$. 
of congruent or symmetric divisions begin with $1,1,2,3,6,9$, $24, \cdots$ The divisions themselves may be classified, for a fixed point, according to the next point in the same subset. We obtain once more the recursion formula leading to $c_{n}=C_{2 n, n} /(n+1)$.

UNIVERSITY OF JERUSALEM

\section{INDEPENDENCE OF RESULTANTS}

TH. MOTZKIN

In this note it is proved that the resultants of $m$ forms, of a sufficiently high degree, in $n$ variables are independent functions of the coefficients of the forms. The proof demands some lemmas on irreducible manifolds, and on monomial manifolds. A monomial manifold is defined by equalities between monomials, that is, products of powers of the coordinates.

In the evaluation of the number of independent hypersurface cross ratios and generalized intersections given in two other notes ${ }^{1} I$ have assumed the above theorem to be true for $2 n-1 \leqq m \leqq 2 n+1$ and, in the case of intersections, where one of the forms is supposed to be linear for $2 n-2 \leqq m \leqq 2 n$.

The resultant $r=r\left(a_{1}, \cdots, a_{n}\right)$ of $n$ forms $a_{k}$ of positive degree $d_{k}$ in $n$ variables $x_{k}$ within an (algebraically) closed field is uniquely defined as an irreducible polynomial in the coefficients of the forms such that $r=1$ if $a_{k}$ is a power of $x_{k}$ and $r=0$ if and only if values $x_{k}$, not all of them 0 , exist for which all $a_{k}=0$. The resultant is almost symmetric, that is, it becomes $r$ or $-r$ if the forms are permuted. The resultant is multiplicative in the sense that if a form $a_{k}$ is a product of forms, then $r$ is the product of the resultants obtained by replacing $a_{k}$ by each of its factors. ${ }^{2}$

THEOREM 1. The $C_{m, n}$ resultants that can be formed of $m$ forms $a_{1}, \cdots, a_{m}$ in $n$ variables are independent functions of the coefficients of the forms, provided that the degree $d_{k}$ of $a_{k}$ exceeds a bound depending only on $k$ and $n$.

Received by the editors September 16, 1946.

${ }^{1}$ The hypersurface cross ratio, Bull. Amer. Math. Soc. vol. 51 (1945) pp. 976-984, \$3.6, and Relations between hypersurface cross ratios, and a combinatorial formula for partitions of a polygon, for permanent preponderance, and for non-associative products, Bull. Amer. Math. Soc. vol. 54 (1948) pp. 352-360.

${ }^{2}$ All these properties of the resultant are well known. Cf. also $\$ 1.1$ of the before mentioned note The hypersurface cross ratio. 